\title{
RAGS: Region-Aided Geometric Snake
}

\author{
Xianghua Xie and Majid Mirmehdi
}

\begin{abstract}
An enhanced, region-aided, geometric active contour that is more tolerant toward weak edges and noise in images is introduced. The proposed method integrates gradient flow forces with region constraints, composed of image region vector flow forces obtained through the diffusion of the region segmentation map. We refer to this as the Region-aided Geometric Snake or RAGS. The diffused region forces can be generated from any reliable region segmentation technique, greylevel or color. This extra region force gives the snake a global complementary view of the boundary information within the image which, along with the local gradient flow, helps detect fuzzy boundaries and overcome noisy regions. The partial differential equation (PDE) resulting from this integration of image gradient flow and diffused region flow is implemented using a level set approach. We present various examples and also evaluate and compare the performance of RAGS on weak boundaries and noisy images.
\end{abstract}

Index Terms-Color snakes, deformable contours, geometric snakes, region segmentation, region-aided snakes, weak-edge leakage.

\section{INTRODUCTION}

D EFORMABLE contour models are commonly used in image processing and computer vision, for example for shape description [1], object localization [2], and visual tracking [3], due to their natural handling of shape variation and independence of operation once initialized. A hypothesised contour, represented as a curve or surface, evolves based on energy minimization methods under the influence of internal forces, external image dependent forces, and certain user-specified constraints, till it converges on the object(s) of interest.

The parametric active contour or "snake," developed by Kass et al. [4] for image segmentation, is attracted toward features such as edges and lines. The energy is composed of terms that control the smoothness of the deforming curve and attract it to the image boundary. Parametric snakes are maintained by a spline, explicitly represented as parameterised curves in a Lagrangian formulation; they have undergone significant improvements since their conception, for example in [5], [6]. Region-based parametric snake frameworks have been reported in [7]-[9], however these still suffer from the disadvantages of parametric contours for shape representation.

The geometric model of active contours, simultaneously proposed by Caselles et al. [10] and Malladi et al. [11], avoids the need to reparameterize the curve and evolves according to

Manuscript received February 14, 2003; revised November 20, 2003. The associate editor coordinating the review of this manuscript and approving it for publication was Dr. Mila Nikolova.

The authors are with the Department of Computer Science, University of Bristol, Bristol BS8 1UB, U.K. (e-mail: xie@cs.bris.ac.uk; majid@cs.bris.ac.uk).

Digital Object Identifier 10.1109/TIP.2004.826124 an Eulerian formulation [12]. Geometric snakes are based on the theory of curve evolution in time according to intrinsic geometric measures of the image and are numerically implemented via level set algorithms [13]. This helps to automatically handle changes in topology and hence, without resorting to dedicated contour tracking, unknown numbers of multiple objects can be detected simultaneously. Furthermore, geometric snakes can have much larger capture areas than parametric snakes.

Whilst geometric or geodesic snakes have been hailed as the solution to the problem of required topological changes during curve evolution [12], they are still prone to shortcomings. These are at least twofold. First, they suffer from leakage into neighboring image regions when confronted with weak edges, and second, they rest at local maximums in noisy images. In this paper, we deal with both of these problems concurrently by introducing a diffused region force into the standard geometric snake formulation. The proposed method is referred to as the Region-aided Geometric Snake or RAGS as it integrates gradient flow forces with diffused region forces. The diffused region force is obtained from the region segmentation map vector flow and gives the snake a global view of the object boundaries. The theory is independent of any particular region segmentation technique which in turn can be generated using different approaches according to the needs of the application at hand. We implement the PDE resulting from the proposed method numerically using level set theory [13], [14] which enables topological changes to be dealt with automatically. We demonstrate the weak edge improvements and evaluate the tolerance to noise as well as present applied results. Using color edge gradients (after [15]), the RAGS snake will be shown to naturally extend to object detection in color images, as also briefly shown in [16].

There has been a number of works based on the geometric snake and level set framework, e.g. [12], [17]-[21]. Siddiqi et al. [17] augmented the performance of the standard geometric snake that minimizes a modified length functional, by combining it with a weighted area functional, with an image dependent weighting factor. This resulted in a modification of the constant term of the curve to help it move in the desired direction more efficiently. However, this still did not provide a satisfactory solution to the weak edge leakage problem [12], [17].

In [20], Chan and Vese described a region-segmentation based active contour that does not use the geometric snake's gradient flow to halt the curve at object boundaries. Instead, this was modeled as an energy minimization of a Mumford-Shah based minimal partition problem and implemented via level sets. Their use of a segmented region map is similar to the concept we have explored here, however their method is constrained to bimodal image segmentation.

$\mathrm{Xu}$ and Prince extended their parametric gradient vector flow (GVF) snake [5] into the Generalized GVF snake (the GGVF) 
in [6]. Later, they also established an equivalence model between parametric and geometric active contours [12] based on the GGVF. The gradient vector flow concept is useful when there are boundary concavities as it preserves the perceptual edge property of snakes [5], [6]. However, the GGVF still has topological problems as will be shown later. Also when a weak edge lies beside a strong edge, the snake is likely to step through toward the strong edge. A geometric GGVF snake enhanced with simple region-based information was presented in [12] and briefly shown to deal with weak edges. However, the examples used were for bimodal regions only. A major benefit of the GVF/GGVF method is the long capture range introduced by the gradient vector diffusion. We apply this concept by diffusing the region vector map and integrating it into the standard geometric snake formulation.

Paragios and Deriche [18], [19] presented a boundary and region unifying geometric snake framework which integrates a specific region segmentation technique with the geometric snake. The Geodesic Active Region model in [18], [19] initially models the image using a Gaussian mixture model to determine the number of regions and their statistics. Then, multiple curves are used to consider each separate homogeneous region and its probabilistically determined boundaries in a bimodal fashion within a geodesic segmentation framework. The geodesic contour model consists of a region boundary term and a region term which acts as the external pressure force in the active region formulation and is implemented via the level set algorithm.

In [21], Yezzi et al. developed coupled curve evolution equations and combined them with image statistics for images of a known number of region types, with every pixel contributing to the statistics of the regions inside and outside an evolving curve. They showed practical examples of bimodal and trimodal region images and discussed a theoretical approach for more than three region classes. More recently, Vese and Chan [22] proposed a different multiple level set representation method to segment an image with more than two regions. Their method needs fewer level set functions to represent the same number of phases and there is no overlap and vacuum in the phases.

This paper is organized as follows. In the next section, we briefly introduce the standard geometric snake and cover some of its associated shortcomings and attempted solutions. In Section III, the region-aided snake RAGS is introduced. We extend RAGS to vector-valued images in Section IV. Implementations, experimental results, and comparisons are presented in Section V. The paper is concluded in Section VI. Weak edge and noise issues are referred to throughout as appropriate.

\section{BACKGROUND AND SHORTCOMINGS}

We briefly review the geometric active contour to help describe the proposed region-aided improvement later. Geometric active contours were introduced by Caselles et al. [10] and Malladi et al. [11] and are based on the theory of curve evolution. Using a reaction-diffusion model from mathematical physics a planar contour is evolved with a velocity vector in the direction normal to the curve. The velocity contains two terms: a constant (hyperbolic) motion term that leads to the formation of shocks from which a representation of shapes can be derived, and a (parabolic) curvature term that smoothes the front, showing up significant features and shortening the curve. In [23] and [24] the formulation of the geodesic active contour, hereafter also referred to as the standard geometric model, was introduced.

Let $C(x, t)$ be a $2 \mathrm{D}$ active contour. The Euclidean curve shortening flow is given by

$$
C_{t}=\kappa \overrightarrow{\mathcal{N}}
$$

where $t$ denotes the time, $\kappa$ is the Euclidean curvature, and $\overrightarrow{\mathcal{N}}$ is the inward unit normal of the contour. This formulation has many useful properties, one of which is that it provides the fastest way to reduce the Euclidean curve length in the direction of the gradient of the curve [25].

Let $I:[0, a] \times[0, b] \rightarrow \Re^{+}$be an input image in which the task of extracting an object contour is considered. The Euclidean length of a curve $C$ is given by

$$
L:=\oint\left|C^{\prime}(q)\right| d q=\oint d s
$$

where $d s$ is the Euclidean arc-length. The standard Euclidean metric $d s^{2}=d x^{2}+d y^{2}$ of the underlying space over which the evolution takes place is modified to a conformal metric $d s_{g}^{2}=$ $g(|\nabla I(C(q))|)^{2}\left(d x^{2}+d y^{2}\right)$ where the term $g($.$) represents a de-$ creasing function such that $g(x) \rightarrow 0$ as $x \rightarrow \infty$, and $g(x) \rightarrow 1$ as $x \rightarrow 0$. Using this metric, a new length definition in Riemannian space is given by

$$
L_{\Re}:=\int_{0}^{1} g(|\nabla I(C(q))|)\left|C^{\prime}(q)\right| d q
$$

Then the steady state of the active contour is achieved by searching for the minimum length curve in the modified Euclidean metric:

$$
\min \int_{0}^{1} g(|\nabla I(C(q))|)\left|C^{\prime}(q)\right| d q
$$

As shown in [23], the steady state is achieved by solving the following equation, showing how each point in the active contour should move in order to decrease the length. The Euler-Lagrange of (4) gives the right-hand side of (5):

$$
C_{t}=g(|\nabla I|) \kappa \overrightarrow{\mathcal{N}}-(\nabla g(|\nabla I|) \cdot \overrightarrow{\mathcal{N}}) \overrightarrow{\mathcal{N}}
$$

Note that (5) has two terms. The first is the curvature term multiplied by the weighting function $g($.$) . In application to shape$ modeling, the weighting factor could be an edge indication function that has larger values in homogeneous regions and very small values on the edges. Since (5) is slow, [23] added a constant inflation term to speed up the convergence. The constant flow is given by $C_{t}=\overrightarrow{\mathcal{N}}$ showing each point on the contour moves in the direction of its normal at a constant speed and on its own can cause a smooth curve to evolve to a singular one. However, integrating it into the geometric snake model lets the curvature flow (1) remain regular:

$$
C_{t}=g(|\nabla I|)(\kappa+c) \overrightarrow{\mathcal{N}}-(\nabla g(|\nabla I|) \cdot \overrightarrow{\mathcal{N}}) \overrightarrow{\mathcal{N}}
$$



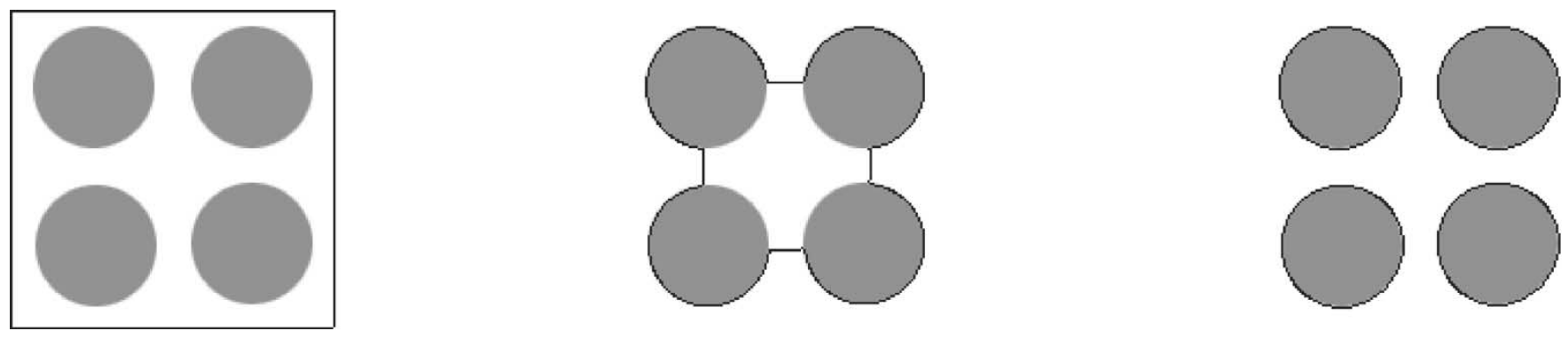

Fig. 1. GGVF snake topological problem—from left: initial snake, final state of the GGVF snake which fails to detect the four objects, and final state of the RAGS snake.

where $c$ is a real constant making the contour shrink or expand to the object boundaries at a constant speed in the normal direction. The second term of (5) or (6) depends on the gradient of the conformal factor $g($.$) and acts like a doublet, which attracts$ the active contour closer to the feature of interest since the vectors of $-\nabla g$ point toward the valleys of $g($.$) , the middle of the$ boundaries. This $-\nabla g$ increases the attraction of the active contour toward these boundaries. For an ideal edge, $g($.) tends to zero. Thus, it tries to force the curve to stop at the edge, but the convergence quality still highly depends on this stopping term. If $g($.$) is not small enough along edges, there will be an under-$ lying constant force caused by $c$.

The geodesic or geometric active contour is numerically implemented using level sets [14], [23], [25].

\section{A. Geometric Snake Problems}

Geometric active contour models have the significant advantage over classical snakes that changes in topology due to the splitting and merging of multiple contours are handled in a natural way. However, they suffer from sensitivity to local minima and only use local information. Hence, they are attracted to noisy pixels and they fail to recognize weaker edges for lack of a better global understanding of the image.

The constant flow term makes the snake expand or shrink. It can speed up the convergence and push the snake into concavities easily when the objects have good contrast, i.e. the gradient values at object boundaries are large. However, when the object boundary is indistinct or has gaps, the snake tends to leak through the boundary mainly because of this constant force. The second term in (6) is designed to attract the contour further close to the object boundary and also to pull back the contour if it leaks through the boundary, but the force may just not be strong enough since it still depends on the gradient values. It can not solve the weak-edge leakage problem. The top row in Fig. 2 demonstrates this shortcoming of the standard geometric snake. The evolving of the snake is based on the gradient information, and as there is gradual change of the intensity, the contour leaks through.

\section{B. Geometric GGVF Snake Problems}

In [5], Xu and Prince presented a new external force for parametric active contours attempting to solve the initialization and convergence problems related to traditional snakes. This external force, which they called gradient vector flow, was computed as a diffusion of the gradient vectors of a greylevel or bi- nary edge map derived from the original image. In [6], the same authors introduced the GGVF, a generalized GVF snake model. GGVF improves over GVF by replacing the constant weighting factor with two spatially varying weighting functions that allows the active contour to converge into long, thin boundary indentations.

Later in [12], Xu et al. showed the GGVF equivalence in a geometric framework. A simple bimodal region force generated as a two-class fuzzy membership function was added to briefly demonstrate weak-edge leakage handling [12]. The GGVF snake is useful when dealing with boundaries with small gaps. However, it's still not robust to weak edges, especially when a weak boundary is close to a strong edge, the snake readily steps through the weak edge and stops at the strong one (illustrated later in Fig. 4).

A further problem with the GGVF snake is that it does not always allow the detection of multiple objects. These topological problems arise, even though the GGVF snake was specified in the geometric model [12], when the vector field is tangent to the snake contour. In such cases there would be no force to push or pull it in the perpendicular direction (to the vectors). This effect is shown in Fig. 1. Both the standard geometric snake and RAGS do not suffer from this problem due to the constant pressure force incorporated in their framework. Note that recently, Paragios et al. [26] also fit the GVF snake into the geometric framework and introduced an adaptive balloon force, which helps the snake expand or shrink when the gradient vector is tangent to the normal of the active contour.

\section{Region-Aided GeOMEtRIC SNAKE}

Here we propose a novel approach to make the geometric snake much more tolerant toward weak edges and image noise. It comprises the integration of the gradient flow forces with diffused region forces in the image resulting in our region-aided geometric snake, RAGS. The gradient flow force supplants the snake with local object boundary information while the region force is based on the global image features. We show that this combination of forces not only improves the performance of the geometric snake toward weak edges, but also makes it more immune to noise. The PDE thus obtained, evolves an initial contour toward final convergence under the influence of both internal forces and boundary-regional image forces, and is implemented via level sets.

The gradient flow force is sensitive to edge information and active contours are generally driven by it, e.g. [4]-[6], [15], [17], 

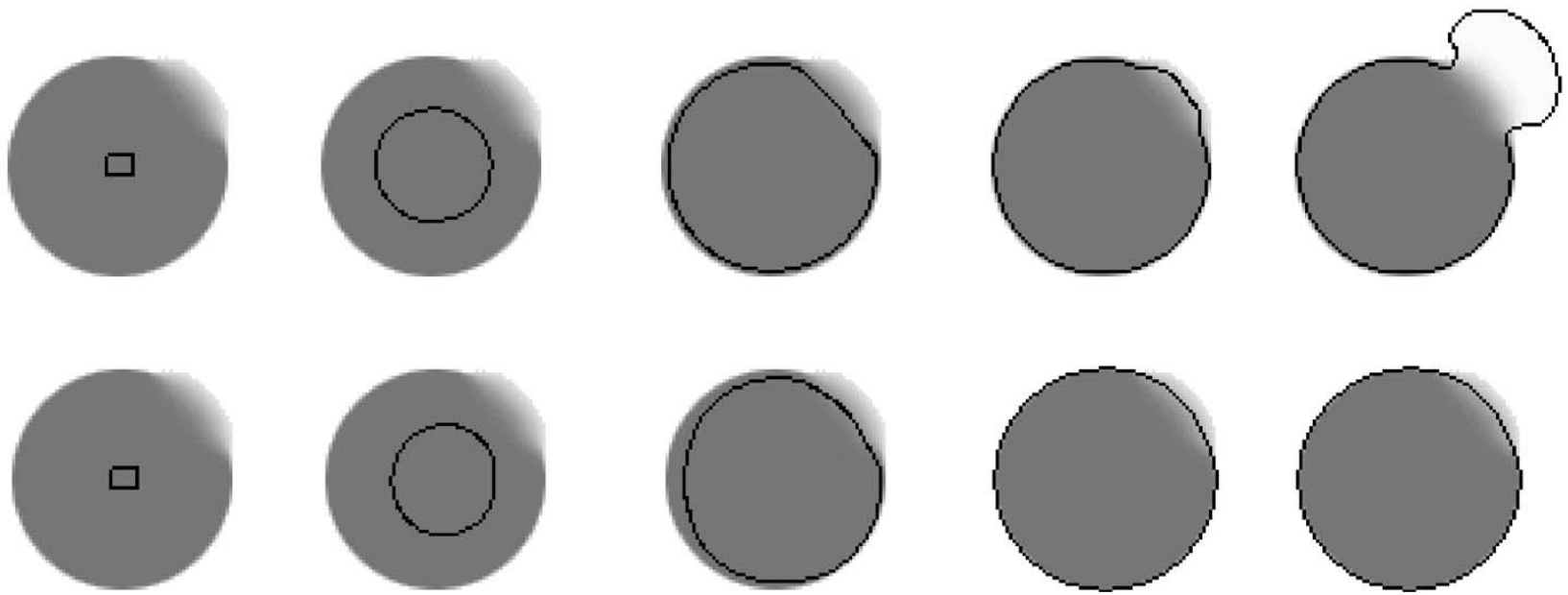

Fig. 2. Weak-edge leakage testing on a synthetic image-top row: geodesic snake steps through; bottom row: RAGS snake converges properly using its extra region force.

[23], [25] to name a few, with one notable exception [20]. The proposed region force can be generated from any image segmentation technique, e.g. [27], [28]. This means that while RAGS is independent of any particular segmentation technique, it is dependent on the quality of the regions produced. However, we show a good degree of tolerance to (reasonable) segmentation quality, and that our snake can indeed also act as a refinement of the results of the initial region segmentation. To examine this, we demonstrate our results on region maps obtained from both the under-segmentation and over-segmentation options of the software from Comaniciu and Meer [27], [29].

\section{A. Region Force Diffusion}

The region force could be generated by a variety of segmentation techniques, greylevel or color. The segmentation splits the image into several regions distinguished by their dominant color. By putting these through a simple gradient filter, we obtain the region boundary map $R$. The magnitude of this region boundary map is then proportional to the color distance between any two adjacent region colors in the $C I E-L^{*} a^{*} b^{*}$ color space. Then, we compute the gradient of the region boundary map $\nabla R$, giving region constraints in the vicinity of the region boundaries. While the snake evolves in a homogeneous image region, it does so mainly based on the gradient flow force. If the snake tries to step from one region into another, it must concur with the region force since it breaks the region criteria, which probably indicates a leakage. The capture area of the pure region force is quite small. A gradient vector diffusion method was proposed in [6] to extend the gradient map vectors further away from the edges for a larger capture field. We use this same concept to diffuse the region boundary gradient map resulting in region forces with a larger capture area along the region boundaries. Hence, we obtain a two dimensional vector field $[\tilde{R}(z)=(u(z), v(z)), z=(x, y)]$ by solving the following equations:

$$
\left\{\begin{array}{l}
p(|\nabla R|) \nabla^{2} u-q(|\nabla R|)\left(u-\nabla R_{u}\right)=0 \\
p(|\nabla R|) \nabla^{2} v-q(|\nabla R|)\left(v-\nabla R_{v}\right)=0
\end{array}\right.
$$

where $\nabla^{2}$ is the Laplacian operator, $p($.$) and q($.$) are weighting$ functions that control the amount of diffusion, and $\nabla R_{u}$ and $\nabla R_{v}$ are the components of vector field $\nabla R$ along the $u$ and $v$ directions. These are selected such that $p($.$) gets smaller as q($. becomes larger with the desirable result that in the proximity of large gradients, there will be very little smoothing and the vector field will be nearly equal to the gradient of the region map. We use the following functions for diffusing the region gradient vectors:

$$
\left\{\begin{array}{l}
p(|\nabla R|)=e^{-\left(\frac{|\nabla R|}{K}\right)} \\
q(|\nabla R|)=1-p(|\nabla R|)
\end{array}\right.
$$

where $K$ is a constant and acts as a trade-off between field smoothness and gradient conformity.

\section{B. Region-Aided Snake Formulation}

Next, we can derive the region-aided geometric snake formulation. The diffused region force is treated as an extra external force of the snake. The original internal and external forces of (6) are given by

$$
\left\{\begin{array}{l}
F_{\text {int }}=g(|\nabla I|) \kappa \overrightarrow{\mathcal{N}} \\
F_{\text {ext }}=g(|\nabla I|) c \overrightarrow{\mathcal{N}}-\nabla g(|\nabla I|)
\end{array}\right.
$$

where $g($.$) is the stopping function as before. Now we add the$ diffused region force $\tilde{R}$ obtained in (7) to the external term:

$$
\left\{\begin{array}{l}
F_{\text {int }}=g(|\nabla I|) \kappa \overrightarrow{\mathcal{N}} \\
F_{\text {ext }}=\alpha g(|\nabla I|) \overrightarrow{\mathcal{N}}+\beta \tilde{R}-\nabla g(|\nabla I|)
\end{array}\right.
$$

where $\alpha$ is a new constant incorporating $c$ and causes behavior that is similar to $c$ in [23] or [15]. Constants $\alpha$ and $\beta$ act as a trade-off between gradient forces and region forces. In practice, constant $\beta$ takes a value in the range $(0,1)$ and depends on the quality of the region segmentation. 
The snake evolves under all the internal and external forces. As only the forces in the normal direction deform the curve, the evolving curve can be represented as

$$
C_{t}=\left[\left(F_{\text {int }}+F_{\text {ext }}\right) \cdot \overrightarrow{\mathcal{N}}\right] \overrightarrow{\mathcal{N}}
$$

Finally, the region-aided geometric snake formulation is

$$
C_{t}=[g(|\nabla I|)(\kappa+\alpha)-\nabla g(|\nabla I|) \cdot \overrightarrow{\mathcal{N}}+\beta \tilde{R} \cdot \overrightarrow{\mathcal{N}}] \overrightarrow{\mathcal{N}}
$$

\section{Level Set Representation}

In this section, we outline the level set representation for the region-aided geometric snake. Level sets describe a moving front and are the basis for the numerical algorithm for curve evolution according to functions of curvature, introduced by Osher and Sethian [13], [14].

Let $C$ be a level set of a function of $\phi:[0, a] \times[0, b] \rightarrow \Re$. That is, $C$ is embedded into the zero level set with $\phi$ an implicit representation of the curve $C$. This representation is parameter free and intrinsic. Given a planar curve that evolves according to $C_{t}=\mathbb{F} \overrightarrow{\mathcal{N}}$ for a given function $\mathbb{F}$, then the embedding function should deform according to $\phi_{t}=\mathbb{F}|\nabla \phi|$, where $\mathbb{F}$ is computed on the level sets. By embedding the evolution of $C$ in that of $\phi$, topological changes of $C$ are handled automatically and accuracy and stability are achieved using the proper numerical algorithm.

The internal curvature and external pressure terms of the RAGS formulation in (12) can be easily transferred to level set representation:

$$
\left\{\begin{array}{l}
C_{t}=g(|\nabla I|) \kappa \overrightarrow{\mathcal{N}} \rightarrow \phi_{t}=g(|\nabla I|) \kappa|\nabla \phi| \\
C_{t}=g(|\nabla I|) c \overrightarrow{\mathcal{N}} \rightarrow \phi_{t}=g(|\nabla I|) c|\nabla \phi|
\end{array}\right.
$$

The external forces in (12) are static vector fields derived from image data which do not change as the active contour deforms. Static force fields are defined on the spatial positions rather than the active contour itself. Since $\overrightarrow{\mathcal{N}}$ is the inward normal, the level set representation of the inward unit normal is given by

$$
\overrightarrow{\mathcal{N}}=-\frac{\nabla \phi}{|\nabla \phi|}
$$

Then we have

$$
\mathbb{F} \cdot \overrightarrow{\mathcal{N}}=-\frac{1}{|\nabla \phi|}(\mathbb{F} \cdot \nabla \phi)
$$

This leads to the level set representation of RAGS as:

$$
\phi_{t}=g(|\nabla I|)(\kappa+\alpha)|\nabla \phi|+\nabla g(|\nabla I|) \cdot \nabla \phi-\beta \tilde{R} \cdot \nabla \phi
$$

where $g($.$) is the stopping function as before. The expression for$ the curvature of the zero level set assigned to the interface itself is given by

$$
\kappa=\operatorname{div}\left(\frac{\nabla \phi}{|\nabla \phi|}\right)=\frac{\phi_{x x} \phi_{y}^{2}-2 \phi_{y} \phi_{x} \phi_{x y}+\phi_{y y} \phi_{x}^{2}}{\left(\phi_{x}^{2}+\phi_{y}^{2}\right)^{\frac{3}{2}}}
$$

\section{REGION-AIDED GEOMETRIC SNAKE ON VECTOR-VALUED IMAGES}

As shown in [23], the theory of boundary detection by the geometric or geodesic snake can be applied to any general "edge detector" function. The stopping function $g$ should tend to zero when reaching edges. Let $f$ be the edge detector. Then, the decreasing function $g$ can be any decreasing function of $f$ such that $g \rightarrow 0$ as $f \rightarrow \infty$. When dealing with graylevel images, the solution (as used in this work) is straight forward:

$$
f=\mid \nabla(\text { Gauss } * I) \mid \text { and } g=\frac{1}{1+f}
$$

We use a similar stopping function for edges obtained directly from vector-valued images such as a color image. We foster the approach developed by di Zenzo [30] which provides a consistent extension of scalar gradients based on a solid theoretical foundation. The concept was also applied in [15] and [31] for their geometric and parametric snakes respectively.

In a vector-valued image the vector edge is considered as the largest difference between eigenvalues in the tensor metric. Let $\Theta\left(u_{1}, u_{2}\right): \Re^{2} \rightarrow \Re^{m}$ be a m-band image for $i=1,2, \ldots, m$. For color images, $m=3$. A point in the image is considered as a vector in $\Re^{m}$. The distance between two points, $P=\left(u_{1}^{0}, u_{2}^{0}\right)$ and $Q=\left(u_{1}^{1}, u_{2}^{1}\right)$, is given by $\triangle \Theta=\Theta(P)-\Theta(Q)$. When this distance tends to the infinitesimal, the difference becomes the differential $d \Theta=\sum_{i=1}^{2}\left(\partial \Theta / \partial u_{i}\right) d u_{i}$ with its squared norm given by

$$
d \Theta^{2}=\sum_{i=1}^{2} \sum_{j=1}^{2} \frac{\partial \Theta}{\partial u_{i}} \frac{\partial \Theta}{\partial u_{j}} d u_{i} d u_{j}
$$

Using standard Riemannian geometry notation, then let $s_{i j}=$ $\left(\partial \Theta / \partial u_{i}\right) \cdot\left(\partial \Theta / \partial u_{j}\right)$, such that

$$
d \Theta^{2}=\sum_{i=1}^{2} \sum_{j=1}^{2} s_{i j} d u_{i} d u_{j}=\left[\begin{array}{l}
d u_{1} \\
d u_{2}
\end{array}\right]^{T}\left[\begin{array}{ll}
s_{11} & s_{12} \\
s_{21} & s_{22}
\end{array}\right]\left[\begin{array}{l}
d u_{1} \\
d u_{2}
\end{array}\right]
$$

For a unit vector $v=(\cos \theta, \sin \theta)$, then $d \Theta^{2}(v)$ indicates the rate of change of the image in the direction of $v$. The extrema of the quadratic form are obtained in the directions of the eigenvectors of the metric tensor $s_{i j}$, and the corresponding eigenvalues are:

$$
\lambda_{ \pm}=\frac{s_{11}+s_{22} \pm \sqrt{\left(s_{11}-s_{22}\right)^{2}+4 s_{12}^{2}}}{2}
$$

with eigenvectors $\left(\cos \theta_{ \pm}, \sin \theta_{ \pm}\right)$where the angles $\theta_{ \pm}$ are given by $\theta_{+}=(1 / 2) \arctan \left(2 s_{12} /\left(s_{11}-s_{22}\right)\right)$ and $\theta_{-}=\theta_{+}+(\pi / 2)$. The maximal and minimal rates of change 

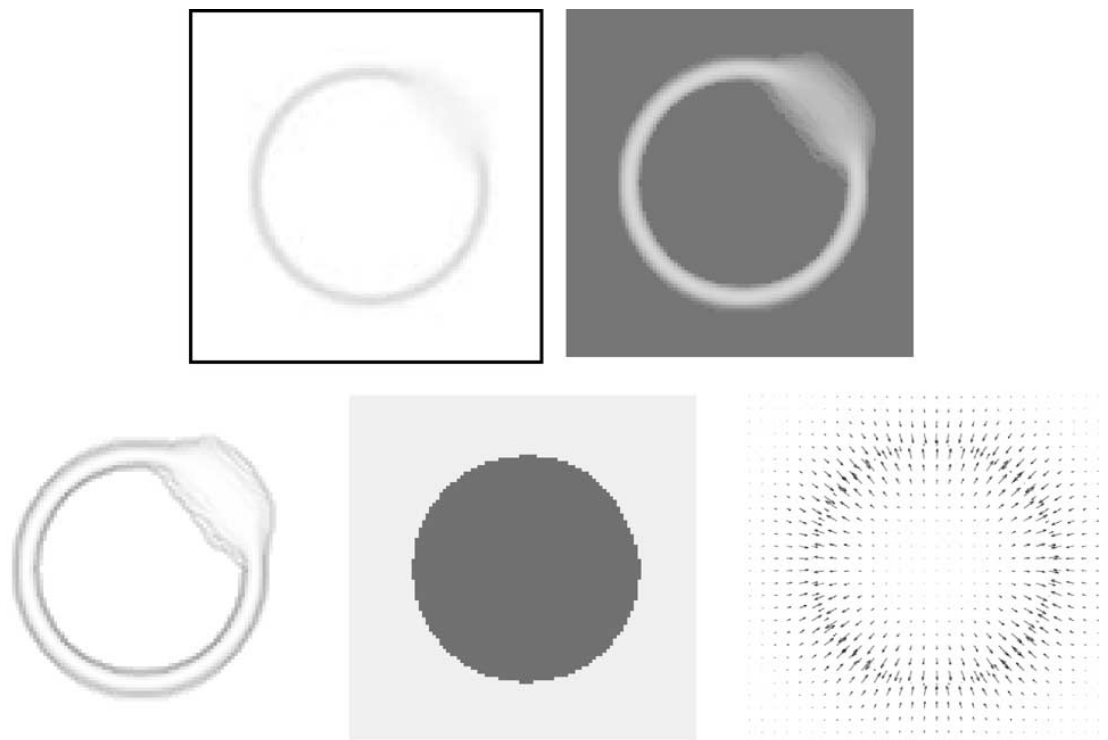

Fig. 3. Diffused region force on weak edge—top row, from left: the edge map and the stopping function $g($.$) of edge map—bottom row, from left: magnitude of$ its gradient $\nabla g($.), the region segmentation map, and the vector map of the diffused region force $\tilde{R}$.

are the $\lambda_{+}$and $\lambda_{-}$eigenvalues respectively, with corresponding directions of change $\theta_{+}$and $\theta_{-}$. The strength of an edge in a vector-valued case is not given simply by the rate of maximal change $\lambda_{+}$, but by the difference between the extremums. Hence, a good approximation function for the vector edge magnitude should be based on $f=f\left(\lambda_{+}, \lambda_{-}\right)$. Now we can extend RAGS to the region-aided geometric color snake by selecting an appropriate edge function $f_{\mathrm{col}}$. The edge stopping function $g_{c o l}$ is defined such that it tends to 0 as $f_{c o l} \rightarrow \infty$. Here, the following functions are used (cf. (18)):

$$
f_{c o l}=\lambda_{+}-\lambda_{-} \text {and } g_{c o l}=\frac{1}{1+f_{c o l}}
$$

Then replacing $g_{\text {col }}($.$) for the edge stopping term g($.$) in (12),$ we have the color RAGS snake:

$C_{t}=\left[g_{c o l}(|\nabla I|)(\kappa+\alpha)-\nabla g_{c o l}(|\nabla I|) \cdot \overrightarrow{\mathcal{N}}+\beta \tilde{R} \cdot \overrightarrow{\mathcal{N}}\right]_{(23)} \overrightarrow{\mathcal{N}}$.

Finally, its level set representation is also given by replacing $g_{\text {col }}($.$) for g($.$) in (16):$

$\phi_{t}=g_{c o l}(|\nabla I|)(\kappa+\alpha)|\nabla \phi|+\nabla g_{c o l}(|\nabla I|) \cdot \nabla \phi-\beta \tilde{R} \cdot \nabla \phi$

\section{EXPERIMENTS AND RESULTS}

In summary, the region-aided geometric (color) snake propagates under the influence of one internal force, i.e. the curvature flow, and three external forces: (a) the pressure force generated by the constant gradient flow, (b) the gradient of the (color) edge stopping force, and (c) the diffused region vector force derived from the region constraints. These constraints can be generated in a variety of ways. In this paper, a general greylevel and color segmentation technique, i.e. the mean shift algorithm [27], [29] is applied. The software made publicly available by the authors of the mean shift algorithm provides standard options for over-segmentation and under-segmentation. We will show results for both of the options.

Furthermore, we present mainly results that show improvements over either the standard geometric snake or the GGVF snake or both, and mainly in images where there are weak edges or noisy regions preventing the aforementioned snakes to perform at their best. Although GGVF's have been reported using greylevel image gradients [6], we apply them to our "color" gradients presented in the previous section, which allows direct comparison with the color RAGS. It must also be noted that the GGVF can sometimes perform better than we have shown in some of the following examples as long as it is initialized differently, i.e much closer to the desired boundary. Unless specifically stated, in all our experiments we have initiated the geometric, GGVF, and RAGS snakes at the same position.

\section{A. Preventing Weak-Edge Leakage}

We first illustrate the way weak-edge leakage is handled on a synthetic image (as in [12], [17]). The test object is a circular shape with a small blurred area on the upper right boundary as shown in Fig. 2. The standard geometric snake steps through the weak edge because the intensity changes so gradually that there is no clear boundary indication in the edge map. The RAGS snake converges to the boundary since the extra diffused region force delivers useful global information about the object boundary and helps prevent the snake from stepping through. Fig. 3 shows, for the test object in Fig. 2, the edge map, the stopping function $g($.$) , magnitude of its gradient \nabla g($.$) , the region$ segmentation map, and the vector map of the diffused region force $\tilde{R}$.

\section{B. Neighboring Weak/Strong Edges}

The next experiment is designed to demonstrate that both the standard geometric snake and the GGVF snake readily step through a weak edge to reach a neighboring strong edge. The test object in Fig. 4 contains a prominent circle inside a fainter 

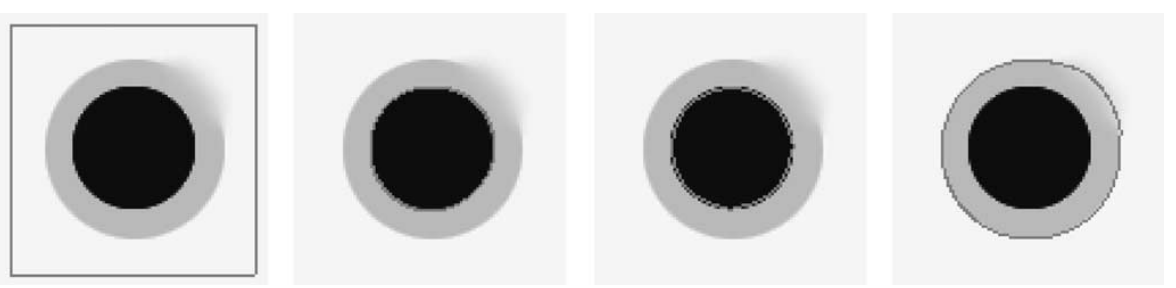

Fig. 4. Strong neighboring edge leakage—-from left: initial snake, geodesic snake steps through weak edge in top right of outer boundary, GGVF is attracted by the stronger inner edge, RAGS snake converges properly using extra region force.
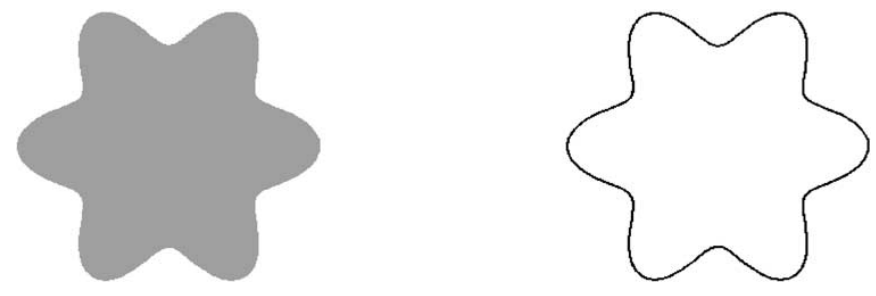

Fig. 5. A shape and its boundary (a harmonic curve).

one. The presence of the weaker edge at the outer boundary is detected only by the RAGS snake. The geodesic snake fails because the weaker outer boundary allows the whole snake to leak through (similar to but in the opposite direction of propagation of Fig. 2). The GGVF snake fails due to the strong gradient vector force caused by the inner object boundary. Practical examples of this can also be observed in Figs. 12 and 13.

\section{Testing on Noisy Images}

We also performed comparative tests to examine for tolerance to noise. For this a Harmonic shape was used, as in [6], shown in Fig. 5. It was generated according to $r=a+b \cos (m \theta+c)$, where $a, b$, and $c$ remain constant and $m$ can be used to produce different numbers of "bumps"; in this case $m=6$. We added varying amounts of noise and measured the accuracy of fit (i.e. boundary description) after convergence. The accuracy was computed using Maximum Radial Error (MRE), i.e. the maximum distance in the radial direction between the true boundary and each active contour.

Impulse noise was added to the original image from $10 \%$ to $60 \%$ as shown in the first column of Fig. 6. The region segmentation data used for RAGS is in the second column (without any post-processing to close gaps etc.). The third, fourth, and fifth columns show the converged snake for the standard geometric, the GGVF, and RAGS snakes respectively. A simple subjective examination clearly demonstrates the superior segmentation quality of the proposed snake. The initial state for the standard geometric and RAGS snakes is a square at the edge of the image, while for the GGVF it is set closer to the true boundary to ensure better convergence. At low percentages of noise, all snakes could find the boundary accurately enough. However, at increasing noise levels $(>20 \%)$, more and more local maximums appear in the gradient flow force field, which prevent the standard geometric and GGVF snakes from converging to the true boundaries. The RAGS snake has a global view of the noisy image and the underlying region force pushes it toward the boundary. The quantitative MRE results are shown in Table I.
TABLE I

MAXIMUM RAdial ERror COMPARISON FOR THE HARMONIC SHAPES IN FIG. 6

\begin{tabular}{c|c|c|c}
\hline$\%$ noise & $\begin{array}{c}\text { Stand. Geom. } \\
\text { Snake Error }\end{array}$ & $\begin{array}{c}\text { GGVF } \\
\text { Snake Error }\end{array}$ & $\begin{array}{c}\text { RAGS } \\
\text { Snake Error }\end{array}$ \\
\hline \hline 0 & 2.00 & 2.00 & 2.00 \\
\hline 10 & 2.23 & 2.24 & 2.00 \\
\hline 20 & 5.00 & 7.07 & 4.03 \\
\hline 30 & 10.00 & 16.03 & 3.41 \\
\hline 40 & 16.16 & 21.31 & 5.22 \\
\hline 50 & 15.81 & 21.00 & 5.38 \\
\hline 60 & 28.17 & 20.10 & 5.83 \\
\hline
\end{tabular}

\section{RAGS on Example Images}

Figs. 7 to 9 demonstrate RAGS in comparison to the standard geometric and GGVF snakes on various greylevel images. Fig. 7 shows a good example of weak-edge leakage at the right side of object of interest. While RAGS does extremely well here, the geometric snake leaks through and the GGVF snake fails to progress in the narrow object at all. In Fig. 8, RAGS achieves a much better overall fit, particularly in the lower regions of the right-hand snake and the upper-right regions of the left-hand snake. In Fig. 9, both RAGS and GGVF manage a better fit than the standard geometric snake at the nose-rest of the glasses, however, the GGVF has been pulled toward a stronger edge at various places, such as the inner side of the mug in the top-right corner of the image. On the other hand, RAGS has stopped at the first outer edge.

Fig. 10 compares the standard geometric snake against the proposed RAGS snake on a color image of a mouth ulcer. A snake is initialized to detect an inner region which has a small blurred section along its upper boundary. This is again a hard case for the geometric snake because the weak edge is difficult to detect without global information. It not only has similar color to the inner and outer areas, but also "dilutes" gradually into the background. Indeed, the standard snake steps through the edge. However, the region-aided geometric snake (last two rows of Fig. 10) reaches its steady state and successfully converges to the inner boundary irrespective of whether the under or oversegmentation of the method in [29] is used to generate the initial region map, as shown.

Fig. 11 demonstrates the improvement over the standard geometric color snake introduced in [15]. Unlike the latter, RAGS manages to ignore the noisy region in the top right of the image and converges tightly around the object, again due to 

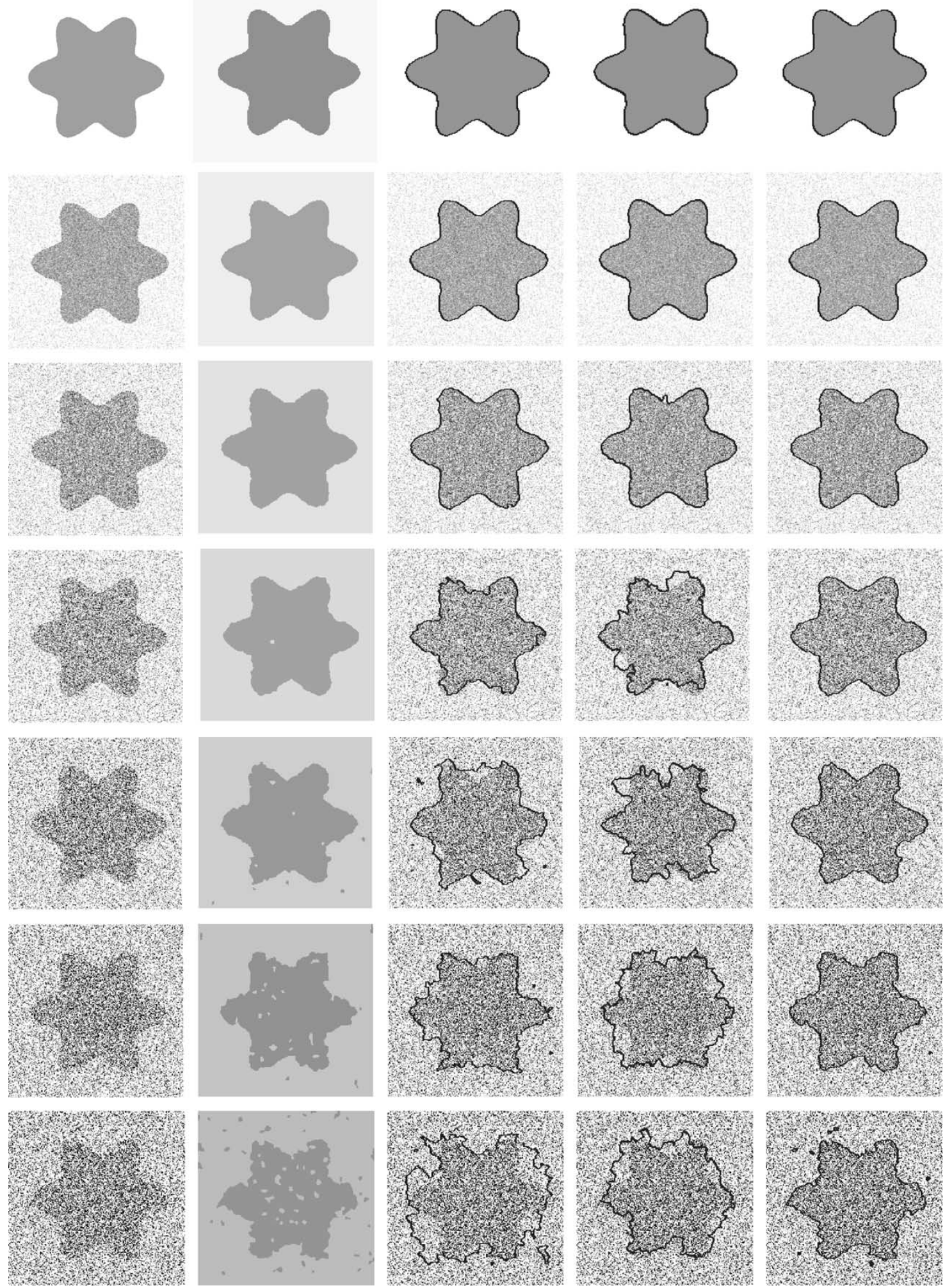

Fig. 6. Shape recovery in noisy images—(column 1) original image with various levels of added Gaussian noise $[0 \%, 10 \%, \ldots, 60 \%]$, (column 2 ) the region maps later diffused by RAGS; (column 3) standard geometric snake results; (column 4) GGVF snake results; (column 5) RAGS results.

the stronger diffused region forces at the object boundary. The GGVF in this example suffers from the problem illustrated in Fig. 1. This GGVF snake also leaks to a stronger inner edge at the bottom-left corner of the lower object (similar to the mug example). This and the next two examples demonstrate the problem shown in Fig. 4. In Fig. 12, a close-up view of a retinal disk is shown after color morphology pre-processing is used to eliminate the blood vessels [2]. The boundary of the optic disk is quite fuzzy and well blended with the background. The region force helps the proposed snake stop at weak edges while the 

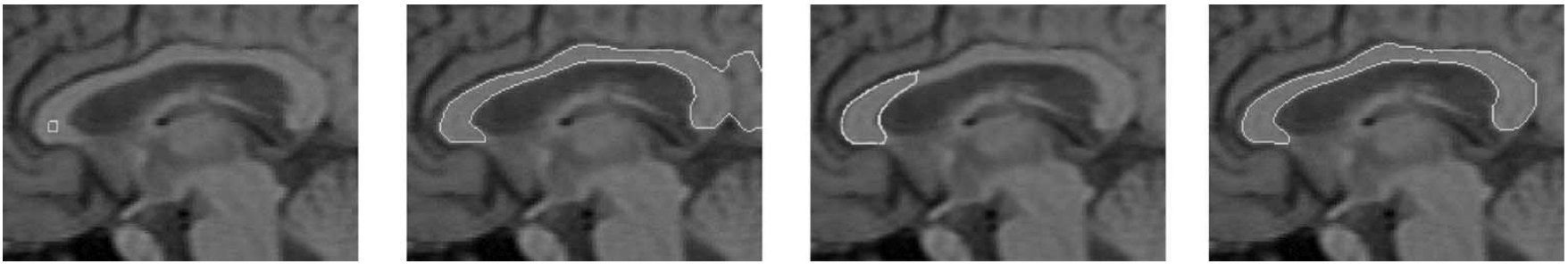

Fig. 7. Brain MRI (corpus callosum) image—from left: Initial snake, standard geometric snake, GGVF snake, and RAGS snake.
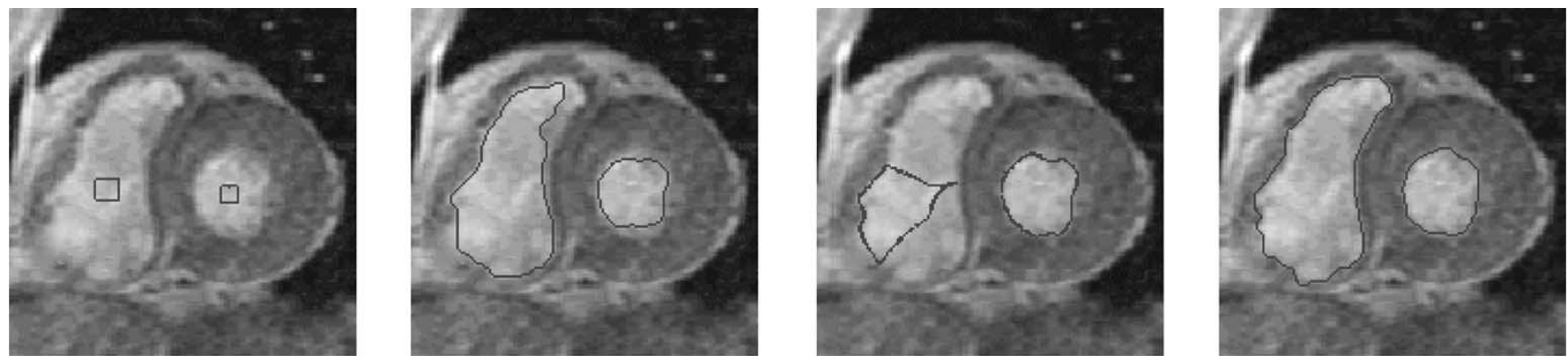

Fig. 8. Heart MRI image — from left: initial snakes, standard geometric snakes, GGVF snakes, and final RAGS snakes showing improvement on the top right of the left snake and the lower region of the right snake.
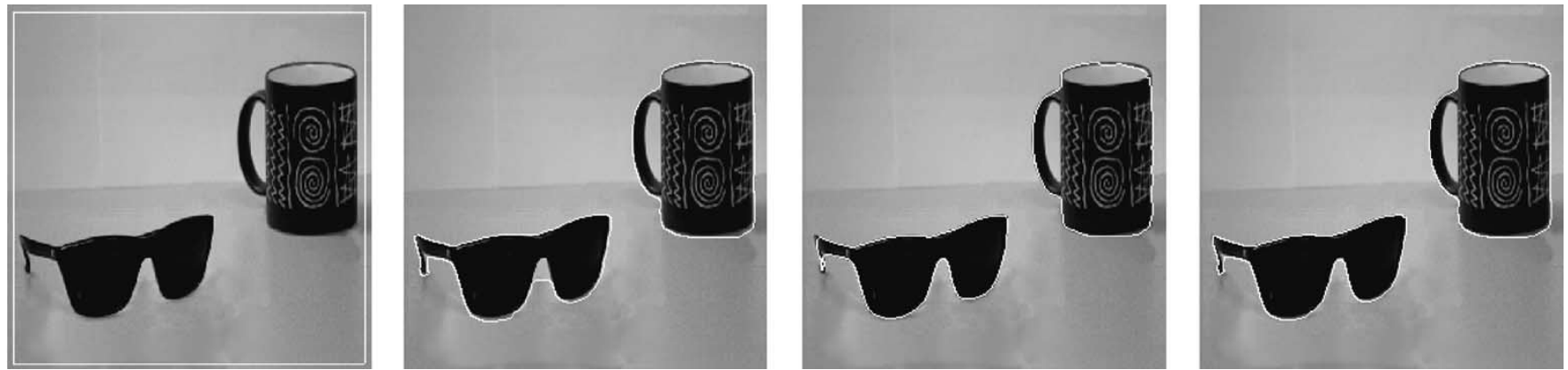

Fig. 9. Mug and sunglasses-from left: initial snake, standard geometric snakes, GGVF snakes, final RAGS snakes showing improvements in the tightness of fit all around, particularly at the sunglasses nose-rest.

standard geometric snake leaks through and the GGVF is highly dependent on where the initial snake is placed (hence GGVF result not provided). Region segmentation results (under and over-segmentation) with corresponding snakes are also shown. In the cell image in Fig. 13, the standard geometric snake splits and converges unsatisfactorily and the GGVF snake is pulled in and out by a stronger inner cell nucleus and neighboring cells respectively, while the RAGS snake converges well to the outer cell boundary without leaking through.

Fig. 14 again shows that RAGS converges on the object very well despite the unsmooth, coarse texture background regions. The standard geodesic snake must deal with local minima of which there are many in the background. Hence, at typical pressure forces it will get stuck in the background and even at larger pressure forces it still is confused by the multiple close edges at the tail of the fish. The GGVF suffers from similar problems. Further examples of RAGS on various images are shown in Fig. 15.
All the examples shown here illustrate the resilience of RAGS to weak edges and noise. However, the RAGS snake does suffer some shortcomings. As with the standard geometric snake, or its color-based relation in [15], or the geometric GGVF snake [12], it will not perform well in highly textured regions in which the gradient flow forces may be hampered by multitudes of texture edge information. It is also dependent on a reasonable segmentation stage, although this was shown to be quite flexible using a popular method with standard built-in options in the software from [29]. Further images and experiments can be found online. ${ }^{1}$

\section{CONCLUSIONS}

A novel method, the region-aided geometric snake or RAGS, was proposed. It integrates the gradient flow forces with region constraints, composed by the image region vector flow forces

${ }^{1}$ http://www.cs.bris.ac.uk/home/xie/rags.htm 

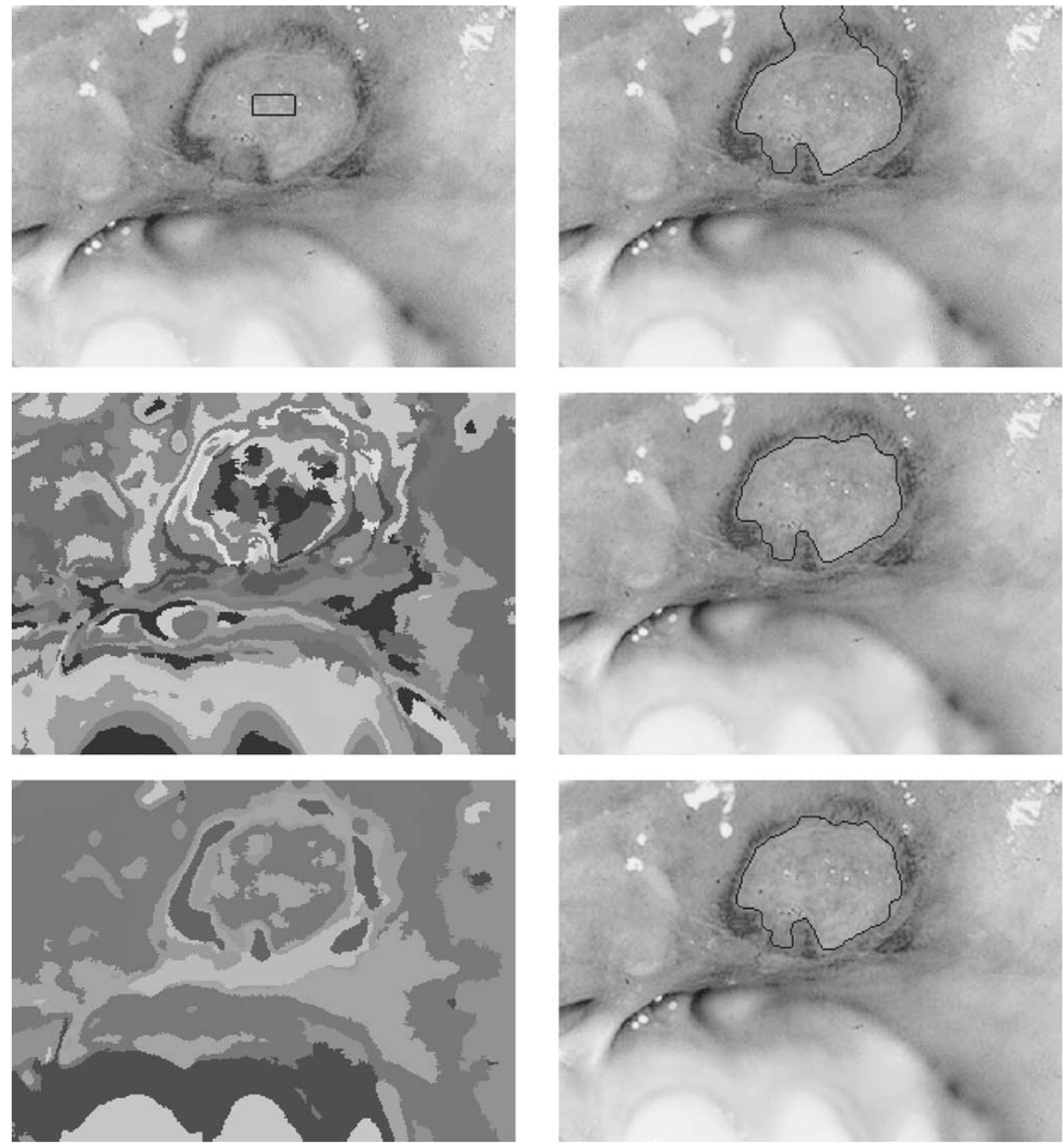

Fig. 10. Weak-edge leakage testing - top row: original image with starting contour and geodesic snake which steps through, middle row: over-segmentation color region map and converged RAGS snake, bottom row: under-segmentation color region map and converged RAGS snake.
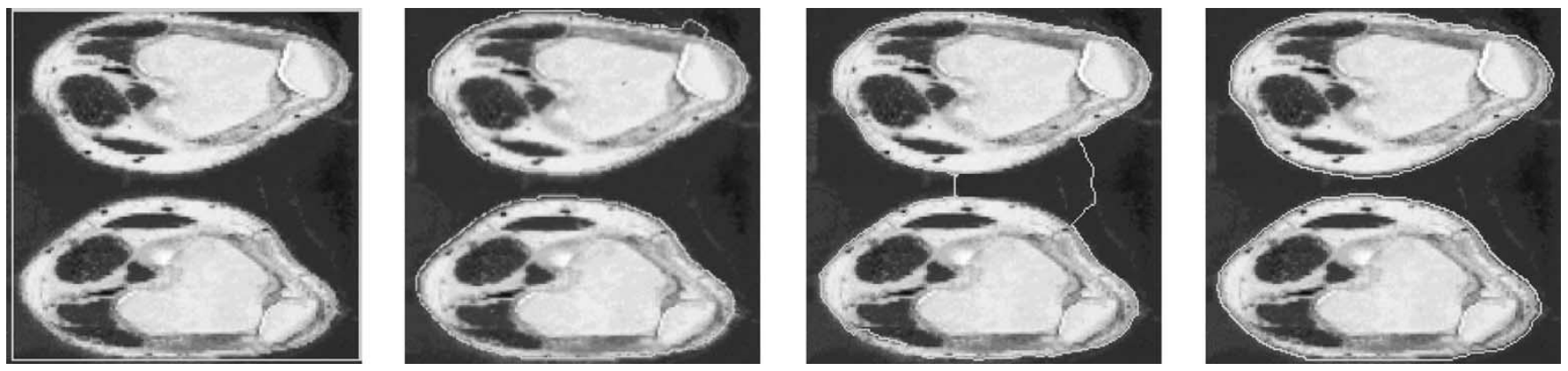

Fig. 11. Thigh slice—from left: initial snake, final Sapiro's geodesic color snakes (from [15]), final GGVF snake, final RAGS snakes.

obtained through the diffusion of the region segmentation map. The theory behind RAGS is stand-alone and hence the region force can be generated from any reasonable segmentation technique. We also showed its simple extension to color gradients. We demonstrated the performance of RAGS, against the standard geometric snake and the geometric GGVF snake, on weak edges and noisy images, as well as on a number of other examples.

The experimental results have shown that the region-aided snake is much more robust toward weak edges. Also it has better convergence quality compared with both the standard geometric snake and the geometric GGVF snake. The weak-edge leakage problem is usually caused by inconclusive edge values at the boundaries, which makes it difficult for gradient-based techniques to define a good edge. The gradual changes do not provide a sufficient minima for the stopping function to prevent the level set accumulating in that area. The diffused region segmentation gives the snake an extra underlying force at the boundaries. It also makes the snake more tolerable to noise as shown by the harmonic shape recovery experiment and many of the real 

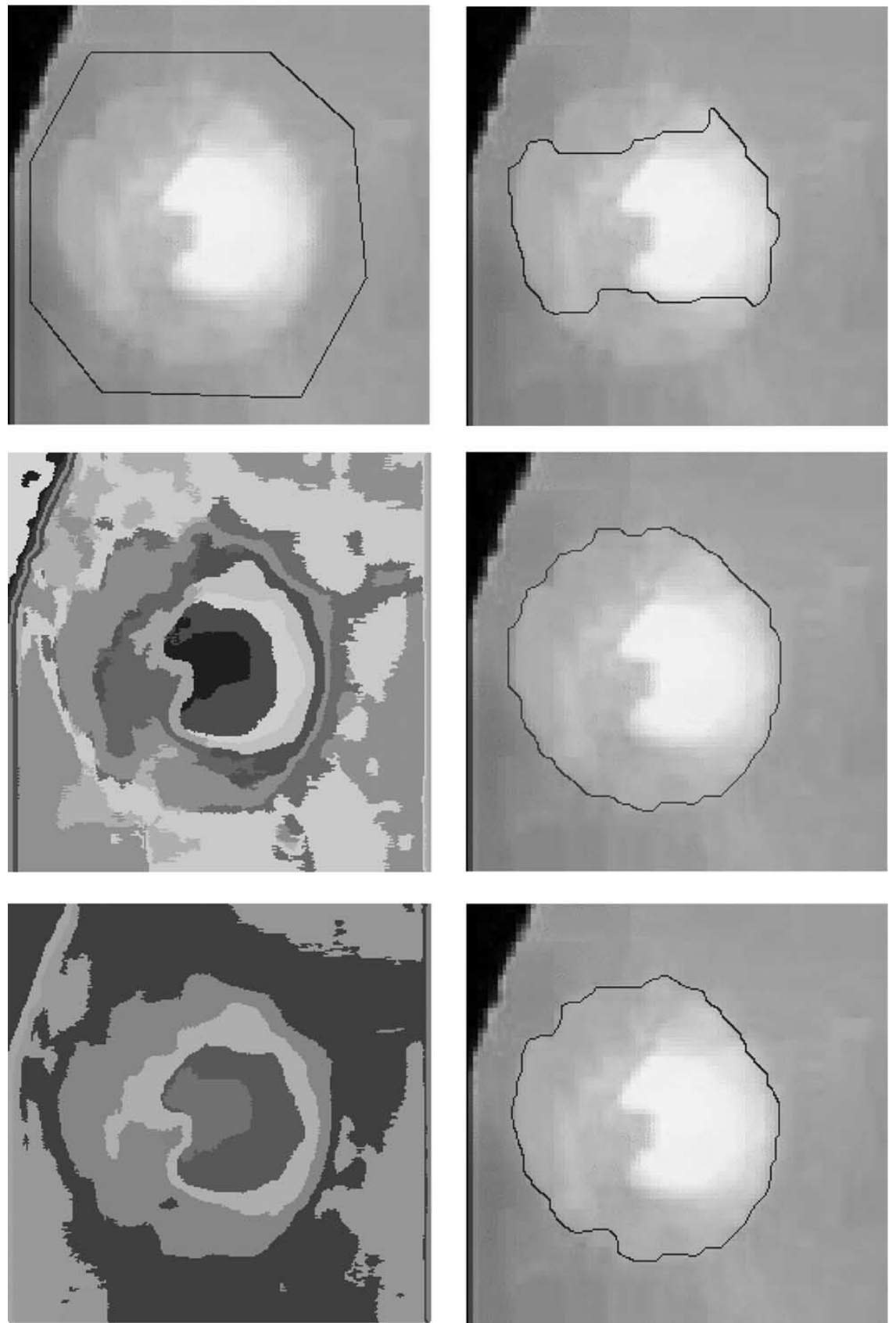

Fig. 12. Optic disk localization - top row: original image with starting contour and geodesic snake which steps through to the stronger central region of the optic disk, middle row: over-segmentation color region map and converged RAGS snake, bottom row: under-segmentation color region map and converged RAGS snake.
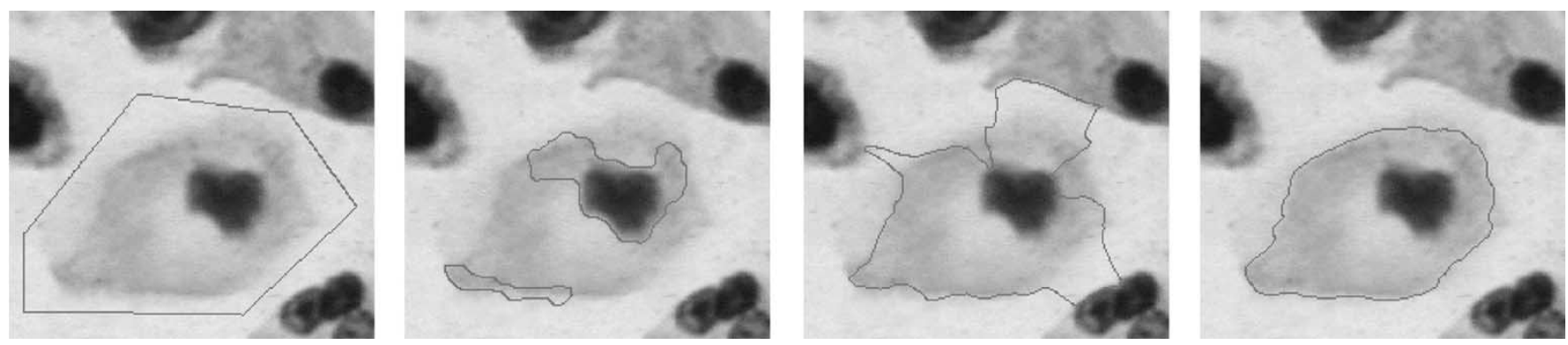

Fig. 13. Cell with strong nucleus feature—from left: initial snake, standard geometric snake, GGVF snake, RAGS snake showing how the stronger inner edge in the cell nucleus does not cause it to lose the outer weaker edge. 

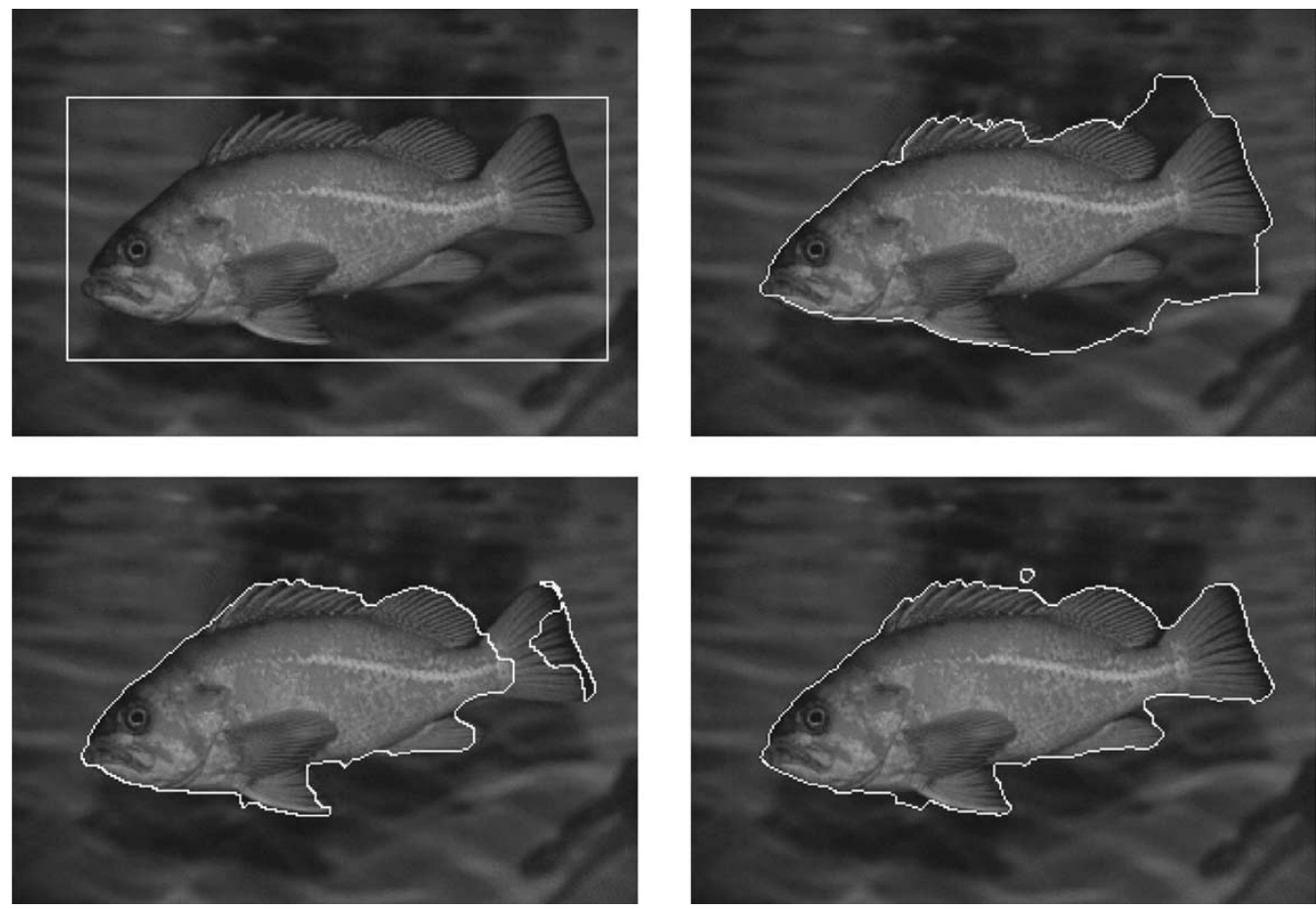

Fig. 14. Fish-top row: initial snake and standard geometric snake, bottom row: GGVF snake and RAGS snake which is not affected by the coarse texture background.
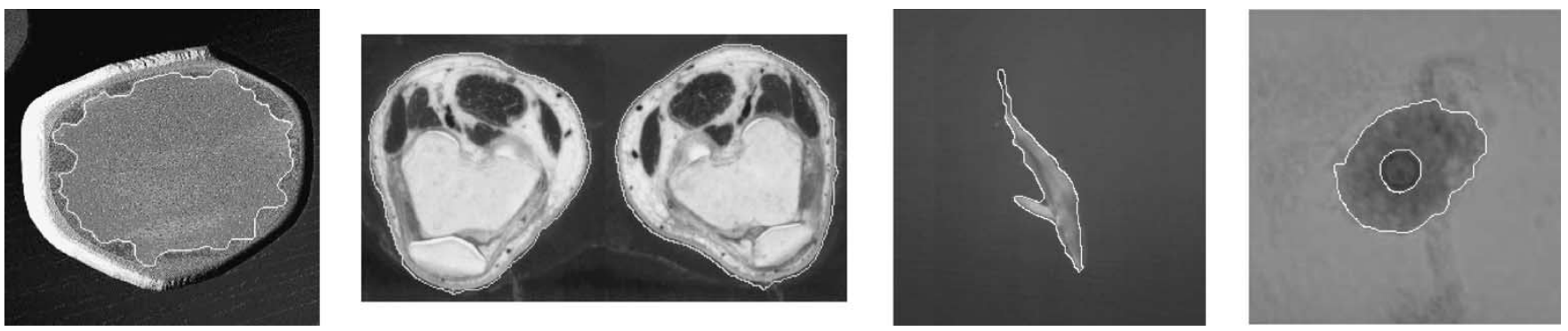

Fig. 15. More RAGS examples.

images. The noise in the image introduces local minima in the stopping function preventing the standard geometric snake to converge to the true boundary. However, for RAGS the diffused region forces give a better global idea of the object boundary in the noise clutter and helps the snake step closer and converge to the global minima.

\section{REFERENCES}

[1] T. Cootes, C. Taylor, D. Cooper, and J. Graham, "Active shape model- their training and application," Comput. Vis. Image Understand., vol. 61, no. 1, pp. 38-59, 1995.

[2] A. Osareh, M. Mirmehdi, B. Thomas, and R. Markham, "Color morphology and snakes for optic disc localization," in Proc. 6th Conf. Med. Image Understand. Anal., 2002, pp. 21-24.

[3] A. Blake and M. Isard, Active Contours. London, U.K.: Springer, 1998.

[4] M. Kass, A. Witkin, and D. Terzopoulos, "Snakes: active contour models," Int. J. Comput. Vis., vol. 1, pp. 321-331, 1988.

[5] C. Xu and J. Prince, "Snakes, shapes, and gradient vector flow," IEEE Trans. Image Processing, vol. 7, no. 3, pp. 359-369, 1998.

[6] — " "Generalized gradient vector flow external forces for active contours," Signal Process., vol. 71, no. 2, pp. 131-139, 1998.
[7] R. Ronfard, "Region-based strategies for active contour models," Int. J Comput. Vis., vol. 13, no. 2, pp. 229-251, 1994.

[8] A. Chakraborty, L. Staib, and J. Duncan, "Deformable boundary finding in medical images by integrating gradient and region information," IEEE Trans. Med. Imag., vol. 15, no. 6, pp. 859-870, 1996.

[9] S. Zhu and A. Yuille, "Region competition: unifying snakes, region growing, and Bayes/MDL for multiband image segmentation," IEEE Trans. Pattern Anal. Machine Intell., vol. 18, no. 9, pp. 884-900, 1996.

[10] V. Caselles, F. Catte, T. Coll, and F. Dibos, "A geometric model for active contours," Numerische Mathematik, vol. 66, pp. 1-31, 1993.

[11] R. Malladi, J. Sethian, and B. Vemuri, "Evolutionary fronts for topology independent shape modeling and recovery," in Proc. 3 rd Eur. Conf. Computer Vision, 1994, pp. 3-13.

[12] C. Xu, J. Yezzi, and J. Prince, "On the relationship between parametric and geometric active contours," in Proc. 34th Asilomar Conf. on Signal, Systems, and Computers, 2000, pp. 483-489.

[13] J. Sethian, Level Set Methods: Evolving Interfaces in Geometry, Fluid Mechanics, Computer Vision, and Materials Science: CUP, 1996.

[14] S. Osher and J. Sethian, "Fronts propagating with curvature-dependent speed: algorithms based on Hamilton-Jacobi formulations," J. Comput. Phys., vol. 79, pp. 12-49, 1988.

[15] G. Sapiro, "Color snakes," Comput. Vis. Image Understand., vol. 68, no. 2, pp. 247-253, 1997.

[16] X. Xie and M. Mirmehdi, "Level-set based geometric color snake with region support," in Proc. ICIP03, 2003, pp. 153-156. 
[17] K. Siddiqi, Y. Lauziere, A. Tannenbaum, and S. Zucker, "Area and length minimizing flows for shape segmentation," IEEE Trans. Image Processing, vol. 7, no. 3, pp. 433-443, 1998.

[18] N. Paragios and R. Deriche, "Coupled geodesic active regions for image segmentation: a level set approach," in Proc. 6th Eur. Conf. on Computer Vision, 2000, pp. 224-240.

[19] — - "Geodesic active regions: a new framework to deal with frame partition problems in computer vision," J. Vis. Commun. Image Represent., vol. 13, no. 1-2, pp. 249-268, 2002.

[20] T. Chan and L. Vese, "Active contours without edges," IEEE Trans. Image Processing, vol. 10, no. 2, pp. 266-277, 2001.

[21] A. Yezzi, A. Tsai, and A. Willsky, "A fully global approach to image segmentation via coupled curve evolution equations," J. Vis. Cотmиn. Image Represent., vol. 13, no. 1-2, pp. 195-216, 2002.

[22] L. Vese and T. Chan, "A multiphase level set framework for image segmentation using the mumford and shah model," Int. Comput. Vis., vol. 50, no. 3, pp. 271-293, 2002

[23] V. Caselles, R. Kimmel, and G. Sapiro, "Geodesic active contour," Int. J. Comput. Vis., vol. 22, no. 1, pp. 61-79, 1997.

[24] R. Malladi, J. Sethian, and B. Vemuri, "Shape modeling with front propagation: a level set approach," IEEE Trans. Pattern Anal. Machine Intell., vol. 17, no. 2, pp. 158-175, 1995.

[25] S. Kichenassamy, A. Kumar, P. Olver, A. Tannenbaum, and A. Yezzi, "Gradient flows and geometric active contour models," in Proc. 5th IEEE Int. Conf. on Computer Vision, 1995, pp. 810-815.

[26] N. Paragios, V. Mellina-Gottardo, and O. Ramesh, "Gradient vector flow fast geodesic active contours," in Proc. ICCV, 2001, pp. 67-73.

[27] D. Comaniciu and P. Meer, "Mean shift analysis and applications," in Proc. 7th IEEE Int. Conf. on Computer Vision, 1999, pp. 1197-1203.

[28] Y. Deng and B. Manjunath, "Unsupervised segmentation of color-texture regions in images and video," IEEE Trans. Pattern Anal. Machine Intell., vol. 23, no. 8, pp. 800-810, 2001.

[29] D. Comaniciu and P. Meer, "Mean shift: a robust approach toward feature space analysis," IEEE Trans. Pattern Anal. Machine Intell., vol. 24, no. 5, pp. 603-619, 2002
[30] S. di Zenzo, "A note on the gradient of a multi-image," Comput. Vis., Graph., Image Process., vol. 33, no. 1, pp. 116-125, 1986.

[31] T. Gevers, S. Ghebreab, and A. Smeulders, "Color invariant snakes," in Proc. 9th British Machine Vision Conf., 1998, pp. 659-670.

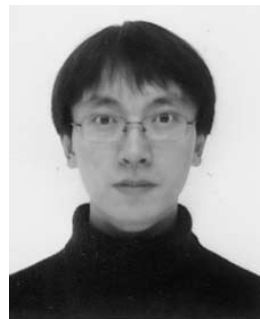

Xianghua Xie received the B.Sc. degree in environmental engineering from the Tongji University, Shanghai, China, in 2000, and the M.Sc. degree in advanced computing from the University of Bristol, U.K., in 2002. He is currently a Ph.D. student and a Research Assistant in the Department of Computer Science, University of Bristol.

His current research interests are texture analysis, texture segmentation, surface inspection, and deformable models.

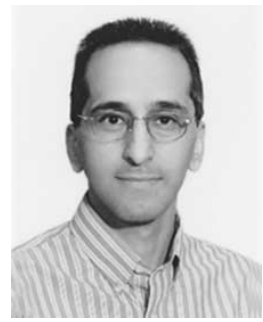

Majid Mirmehdi received the B.Sc. (Hons) and Ph.D. degrees in computer science in 1985 and 1991, respectively, from the City University, London, U.K.

$\mathrm{He}$ has worked both in industry and in academia. He was employed in 1997 as a Lecturer in the Department of Computer Science at Bristol University, U.K., and since 2002, as a Senior Lecturer. His research interests are in image processing, computer vision, pattern recognition, and parallel processing.

Dr. Mirmehdi has co-chaired and co-organized several conferences and has published over 80 papers. He is a member of the IEE. He is also a member of the Executive Committee of the British Machine Vision Association and the Publicity Officer of the BMVA. 